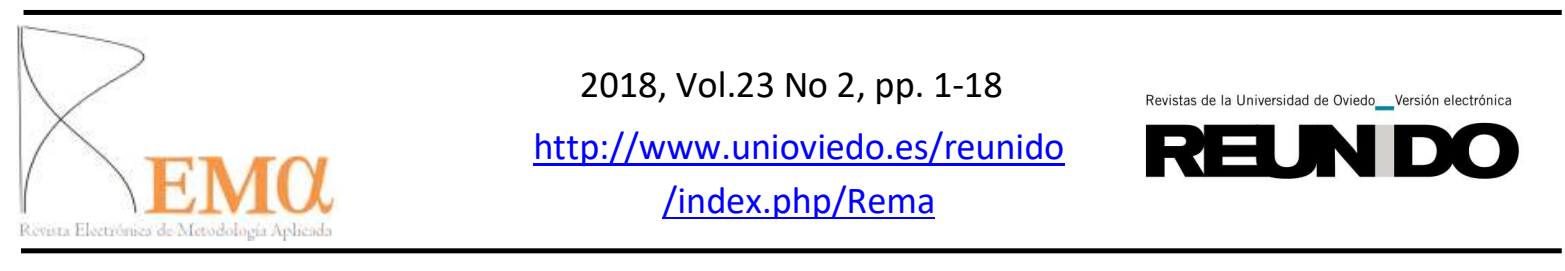

¿Cómo influye el tamaño del campo de texto en las respuestas a preguntas

abiertas?

Evidencias de un diseño experimental.

The influence of box size on responses to open-ended questions: Results from a split-ballot experiment. Carmen María León ${ }^{1}$, Eva Aizpurúa ${ }^{2}$ y David Vázquez ${ }^{1}$

${ }^{1}$ Centro de Investigación en Criminología, Universidad de Castilla-La Mancha ${ }^{2}$ Centre for Social \& Behavioral Research, University of Northern Iowa.

\title{
RESUMEN
}

El diseño visual de los cuestionarios puede afectar a la calidad de los datos obtenidos, especialmente cuando se formulan preguntas abiertas donde los encuestados responden con sus propias palabras. En este trabajo se analizan los efectos de manipular el tamaño del espacio proporcionado para la respuesta en un conjunto de preguntas abiertas incluidas en un cuestionario auto-administrado sobre opiniones hacia la administración de justicia en España. Para ello se recurrió a un experimento split-ballot, dividiendo la muestra $(N=100)$ en dos mitades equivalentes que recibieron dos cuestionarios con el mismo contenido, pero con diferentes tamaños de campo de respuesta (pequeño y grande) en 16 preguntas abiertas. Los resultados muestran que los participantes que recibieron campos de texto grandes escribieron un mayor número de palabras en sus respuestas. Sin embargo, la manipulación en el campo de texto no influyó en 1) el número de temas abordados; ni en 2) el tiempo empleado para cumplimentar los cuestionarios. Sobre la base de estos resultados se discuten las implicaciones para el diseño de cuestionarios.

Palabras clave: Opinión pública, encuestas, preguntas abiertas, diseño visual.

\begin{abstract}
The visual design of questionnaires can affect the quality of the data obtained, especially when asking open-ended questions that respondents answer in their own words. In this paper, we analyze the effects of manipulating the size of the text boxes provided for answers to a set of open-ended questions in a self-administered questionnaire about opinions of the Criminal Justice system in Spain. For this, a split-ballot experiment was conducted dividing the sample $(N=100)$ into two equivalent halves. One half received questionnaires with small box sizes for the answers to the 16 open-ended questions while the other half received questionnaires with larger box sizes. The content on the questionnaires was the same. The results showed that those participants who received larger text boxes provided longer answers. However, manipulation of the text box did not influence 1) the number of issues addressed; or 2) response times. The results and their implications for questionnaire design are discussed.
\end{abstract}

Keywords: Public opinion, surveys, open-ended questions, visual design.

Contacto: Carmen.Leon@uclm.es

Agradecimientos: Agradecemos a los editores y a los revisores anónimos de REMA las aportaciones realizadas que han contribuido a mejorar la claridad y calidad científica del trabajo. 


\section{1.- Introducción}

Los hallazgos de la literatura especializada sugieren que el diseño visual de los cuestionarios, en general, y el diseño visual de las preguntas, en concreto, afectan a las respuestas obtenidas en cuestionarios auto-administrados (Christian, Dillman, \& Smyth, 2007; Couper, Conrad, \& Tourangeau, 2007; Dillman, Smyth, \& Christian, 2009). En este sentido, se ha observado que las características visuales de las preguntas influyen sobre cómo los participantes interpretan su contenido, las procesan y cómo deciden expresar sus respuestas (Groves et al., 2009; Tourangeau, Couper, \& Conrad, 2004). Sin embargo, estos estudios han analizado principalmente el efecto que ejerce el diseño visual de las preguntas cerradas en las respuestas obtenidas, manipulando la ubicación de las alternativas de respuesta, el espacio existente entre ellas o las imágenes que acompañan a las preguntas (Couper et al., 2007; Tourangeau et al., 2004). Las preguntas abiertas, en cambio, han recibido una menor atención en la literatura y el conocimiento acumulado es menor. A pesar de ello, recientes estudios han puesto de relieve la importancia que tiene el tamaño del campo de texto ${ }^{1}$ en este tipo de preguntas, pues proporciona información a los encuestados sobre las expectativas que el investigador tiene acerca del tipo, la estructura o la extensión de las respuestas deseadas (Mohr, Sell, \& Lindsay, 2015), lo que puede condicionar las respuestas de los encuestados. Considerando la escasa literatura previa en este ámbito, el objetivo principal de este trabajo consiste en examinar cómo afecta el diseño visual de las preguntas abiertas, concretamente el espacio proporcionado para responder, a las respuestas que ofrecen los participantes a una serie de preguntas incluidas en una encuesta auto-administrada en papel.

\section{1.- Las Preguntas de Respuesta Abierta}

Una de las primeras decisiones que el investigador debe tomar al diseñar un cuestionario es el tipo de preguntas que va a incluir en el mismo. Las preguntas abiertas son aquellas que permiten a los encuestados responder con sus propias palabras (Krosnick \& Presser, 2009). Atendiendo al tipo de respuesta requerida, éstas pueden ser de naturaleza narrativa o simplemente precisar la enumeración de palabras o elementos numéricos. En el primer caso se habla de preguntas descriptivas, mientras que en el segundo y tercer caso se habla de preguntas de listado y preguntas de respuesta numérica, respectivamente.

Las preguntas descriptivas suelen demandar al encuestado la descripción y/o explicación de una situación, un concepto, una persona... en base a sus propias observaciones o a la información que posee al respecto. Una de las características propias de las preguntas de naturaleza descriptiva es que requieren un buen nivel de redacción y una cantidad mínima de texto en la respuesta para que ésta sea óptima y legible. Un ejemplo de este tipo de preguntas lo encontramos en la Encuesta a Víctimas en España (Díez-Ripollés \& García-España, 2009), donde se preguntaba: “¿Por qué motivo se produjo el último contacto con la Policía?”, dejando que el encuestado respondiese libremente explicando el motivo por el que se produjo ese contacto.

A diferencia de las preguntas de naturaleza descriptiva, las preguntas de tipo listado requieren para su respuesta un nivel de redacción menor. Este tipo de preguntas pueden responderse a través de una frase corta o mediante un listado de palabras. Por ejemplo, en los barómetros del Centro de Investigaciones Sociológicas suele preguntarse: “¿Cuál es, a su juicio, el principal problema que existe actualmente en España?, ¿Y el segundo?, ¿Y el tercero?”. Para responder, basta con una mera enumeración de problemas, sin necesidad de elaborar enunciados largos y complejos. 
Por último, en las preguntas de respuesta numérica el resultado ha de ser uno o más números. Dentro de este tipo de preguntas se encuentran aquellas que preguntan por la edad, la fecha de nacimiento, el número de teléfono, el código postal o el número de hijos en el hogar. Normalmente, la respuesta a este tipo de preguntas se considera sencilla; sin embargo, incluir cuestiones de esta naturaleza en los cuestionarios supone cierto riesgo para el investigador, ya que, a pesar de no ser un problema exclusivo de las preguntas de respuesta numérica, especialmente este tipo de preguntas pueden dar como resultado respuestas inexactas o incluso generar valores inválidos cuando los encuestados no se ajustan al formato requerido (Couper, Kennedy, Conrad, \& Tourangeau, 2011). No obstante, las preguntas de esta naturaleza son muy recurrentes, especialmente en el bloque de preguntas sociodemográficas.

\section{2.- Los Efectos del Tamaño del Campo de Texto en las Respuestas de los Encuestados a Preguntas Abiertas}

La literatura previa en el ámbito de los efectos del tamaño del campo de respuesta ha llegado principalmente a tres conclusiones:

1. Los campos de texto grandes generan respuestas más largas en comparación con los campos de texto pequeños (Christian \& Dillman, 2004; Israel, 2010; Smyth, Dillman, Christian, \& McBride, 2009; Stern, Dillman, \& Smyth, 2007).

2. Los tamaños grandes del campo de texto suelen generar un desarrollo más variado de temas que los tamaños pequeños del campo de texto (Chaudhary \& Israel, 2016; Christian \& Dillman, 2004; Israel, 2006; Smith, 1993).

3. Los campos de texto grandes suelen generar un mayor número de respuestas en blanco en comparación con los campos de texto pequeños (Keusch, 2014; Scholz \& Zuell, 2012; Zuell, Menold, \& Körber, 2014).

En relación con la primera de las conclusiones, el efecto del tamaño del campo de texto sobre la extensión de la respuesta, Christian y Dillman (2004) encontraron que el mayor de los dos tamaños del campo de respuesta empleados en una encuesta autoadministrada en papel (Paper-and-Pencil, PAP) entre estudiantes universitarios resultó en un mayor número de palabras en las respuestas. Posteriormente, estos hallazgos fueron corroborados por Stern et al. (2007) utilizando los datos de una encuesta online. Por su parte, Smyth et al. (2009) encontraron que el efecto del tamaño del campo de texto sobre la extensión de las respuestas estaba mediado por el momento en el que los encuestados respondían al cuestionario. Así, los autores hallaron que en el caso de los encuestados denominados "precoces"2 el campo de texto grande resultó en un mayor número de palabras que el campo de tex to pequeño. Sin embargo, esto no ocurrió con los encuestados "tardíos". Además, encontraron que, en comparación con las encuestas autoadministradas en papel, las encuestas online producían un mayor número de palabras en su respuesta cuando el tamaño del campo de texto era más grande, con independencia de que fuesen encuestados "tardíos" o "precoces". Más recientemente Israel (2010), en consonancia con los hallazgos descritos anteriormente, descubrió un aumento lineal entre el número de palabras y el tamaño del campo de respuesta.

Tomados en conjunto, todos estos estudios proporcionan una base sólida para afirmar que un mayor tamaño del campo de respuesta en preguntas abiertas actúa como un indicador visual para los encuestados acerca de la cantidad de información esperada por los investigadores (Chaudhary \& Israel, 2016). Además, estos hallazgos reflejan que los participantes no sólo utilizan el contenido de los enunciados de las preguntas a la hora de guiar sus respuestas, sino que éstas se encuentran también condicionadas por indicadores visuales, siendo éstos tan importantes como los primeros a la hora de diseñar cuestionarios. Así, atendiendo a las consideraciones precedentes, en el presente trabajo 
se ha planteado la hipótesis de que las respuestas proporcionadas a preguntas con campos de texto más grandes constarán de un mayor número de palabras que las respuestas proporcionadas a preguntas con campos de texto comparativamente más pequeños.

En lo que se refiere a la segunda de las conclusiones, referida al efecto del tamaño del campo de texto sobre la variedad de temas que se abordan en las respuestas, Christian y Dillman (2004), en una encuesta auto-administrada en papel entre estudiantes universitarios, encontraron que los tamaños de texto grandes resultaron en un mayor número de temas en las respuestas que los tamaños de texto pequeños. En sintonía con estos resultados, Israel (2006) concluyó que el tamaño del campo de texto grande sugería al entrevistado que el investigador deseaba más información que cuando se empleaban campos de texto pequeños, lo que conducía al encuestado a elaborar respuestas con un mayor número de temas. Sin embargo, estos resultados han demostrado no ser concluyentes. De este modo, Chaudhary e Israel (2016) encontraron que el incremento del número de temas en los cuestionarios que empleaban campos de texto grandes respondía a una combinación de factores entre los que se encontraban, además del tamaño del campo de respuesta, los comentarios motivacionales y aquellos que destacaban la importancia de la pregunta. En consecuencia y como indicó Smith (1993), que los tamaños del campo de texto grandes sugieran respuestas más largas no significa que los encuestados aborden un mayor número de temas en sus respuestas, ya que estos podrían estar empleando un mayor número de palabras para proporcionar mayor información acerca de un mismo tema. Considerando toda esta evidencia acumulada, esta investigación parte de la hipótesis de que las respuestas obtenidas en preguntas con campos de texto más grandes abordarán un mayor número de temas que las respuestas recogidas en preguntas con campos de texto más pequeños.

Por último, es preciso señalar que uno de los aspectos que más preocupa a los investigadores en relación con las preguntas abiertas son las respuestas en blanco, especialmente cuando se trata de cuestionarios auto-administrados donde el entrevistador no puede facilitar la respuesta repitiendo la pregunta, por ejemplo. Dentro de este ámbito, en lo que concierne a la tercera de las conclusiones formuladas al inicio de este apartado, mayoritariamente se ha sustentado la idea de que los campos de texto grandes generarán un aumento de las respuestas en blanco en comparación con los campos de texto pequeños. Los autores han justificado estos hallazgos atendiendo a la sobrecarga que recae sobre los encuestados al asociar la necesidad de una respuesta más larga con el tamaño del campo de respuesta más grande, lo que podría disuadirlos de proporcionar una respuesta en mayor medida que si el campo de texto fuese de menor tamaño. Sin embargo, la literatura muestra que los hallazgos no son consistentes al respecto. En este sentido, si bien diversos estudios que han utilizado cuestionarios auto-administrados han encontrado que el tamaño del campo de texto no afecta al número de respuestas en blanco (Christian \& Dillman, 2004; Emde \& Fuchs, 2012; Israel, 2006, 2010; Smyth et al., 2009), estos resultados, como se verá más adelante, parecen no reproducirse en las encuestas online, donde diversos autores (Keusch, 2014; Scholz \& Zuell, 2012; Zuell et al., 2014) han encontrado un aumento significativo en el número de respuestas en blanco cuando se emplean tamaños de texto grandes. Sobre la base de los hallazgos que han empleado cuestionarios auto-administrados, en el estudio que se presenta, se ha formulado la hipótesis de que el tamaño de los campos de texto no afectará al número de respuestas en blanco.

Por otra parte, a pesar de no contar con apoyo teórico o empírico que lo sustente, se ha planteado una cuarta hipótesis fruto del razonamiento lógico de que un mayor número de palabras y un mayor número de temas en las respuestas, devendrá en un mayor tiempo empleado para cumplimentar el cuestionario. En este sentido se ha hipotetizado 
que los campos de texto grandes aumentarán el tiempo medio de cumplimentación del cuestionario en comparación con los campos de texto más pequeños.

\section{3.- Factores Relacionados con el Efecto del Tamaño del Campo de Texto en las Respuestas de los Encuestados a Preguntas Abiertas}

Estudios previos han encontrado que los efectos del tamaño del campo proporcionado para la respuesta pueden verse influidos, a su vez, por otros factores. Entre algunos de los que han generado hallazgos más consistentes se encuentran: el tipo de preguntas con respecto a su naturaleza (descriptivas, de listado o de respuesta numérica), los procedimientos de administración del cuestionario (online o en papel) y las características de los participantes, donde se incluye el interés de los encuestados en el tema del cuestionario.

En cuanto a la naturaleza de las preguntas, la literatura acumulada ha mostrado que este constituye un aspecto clave a la hora de explicar los efectos del tamaño de los campos de texto sobre las respuestas. De este modo, los estudios han encontrado que el tamaño del campo de texto influye especialmente en la cantidad de información que los encuestados proporcionan a preguntas que requieren una respuesta descriptiva, mientras que las preguntas de listado o aquellas que pueden responderse a través de elementos numéricos parecen verse menos afectadas por el tamaño del campo de respuesta proporcionado (Couper et al., 2011; Keusch, 2014). Sobre la base de estos hallazgos se ha establecido la hipótesis de que las preguntas descriptivas se verán más afectadas por el tamaño del campo de respuesta que las preguntas de listado.

Por otro lado, con la creciente popularidad de las encuestas multi-modo y la diversificación de los procedimientos de recogida de datos, se ha encontrado que los efectos de las manipulaciones en el diseño visual de los cuestionarios pueden diferir dependiendo de los procedimientos elegidos para administrar los cuestionarios (Mohr et al., 2015). En este sentido, como se apuntaba en párrafos anteriores, diversos estudios han encontrado que las preguntas abiertas en las encuestas online generan menos respuestas en blanco y contestaciones más largas, con un mayor número de temas, que las encuestas auto-administradas en papel (Barrios, Villarroya, Borrego, \& Ollé, 2010; Deutskens, de Ruyter, \& Wetzels, 2006; Hedberg, Wallace, \& Cesar, 2013; Kwak \& Radler, 2002; MacElroy, Mikucki, \& McDowell, 2002). MacElroy, Mikucki y McDowell (2002) atribuyen esta diferencia a la mayor limitación de espacio en las encuestas autoadministradas en papel y a una escritura más rápida gracias al teclado, que facilita la impresión de pensamientos en comparación con la escritura manual. Por su parte, el estudio realizado por Fuchs (2009), donde se manipulaba experimentalmente el tamaño de los campos de respuesta en una serie de preguntas numéricas tanto en una encuesta en papel como en una encuesta online, encontró diferencias significativas en la longitud de las respuestas entre los distintos tamaños del campo de texto empleados (grandes versus pequeños) en la versión en papel del cuestionario, pero no en la versión online. En consecuencia, parece que la administración online del cuestionario mitiga los efectos del tamaño del campo de texto en la longitud de las respuestas cuando se compara con la administración del mismo en papel.

Investigaciones previas también han examinado el efecto de ciertas variables sociodemográficas como son el sexo de los encuestados, su edad y nivel educativo, en las características de sus respuestas (Denscombe, 2008; Israel, 2010; Stern et al., 2007). Estos estudios han hallado que los encuestados mayores de 60 años, las mujeres y los encuestados que tenían formación académica universitaria proporcionaban respuestas más largas que otros grupos cuando les fueron proporcionados campos de texto grandes (Stern et al., 2007). Denscombe (2008), en una muestra con jóvenes de entre 15 y 16 años, 
encontró que el sexo y las expectativas educativas influían en la longitud de la respuesta a preguntas abiertas. Además, el estudio realizado por Israel (2010) evidenció que las mujeres y aquellos encuestados con mayor formación académica tenían más probabilidades de escribir fuera del espacio destinado a la respuesta cuando se les proporcionaban tamaños pequeños del campo de texto.

Dentro de las características de los encuestados puede incluirse también el interés de éstos en el tema del cuestionario. La evidencia previa muestra que el interés personal en el tema puede tener una influencia sustancial en la respuesta de los encuestados, independientemente de los tamaños del campo de texto (Keusch, 2014). En este sentido, Holland y Christian (2009) concluyeron que la tasa de respuesta a preguntas abiertas se incrementaba cuando los encuestados estaban interesados en el tema. Smyth et al. (2009), en una encuesta online con muestra universitaria, encontraron que el énfasis transmitido a los encuestados sobre la importancia de una pregunta abierta aumentaba el número de palabras y el número de temas elaborado por los encuestados en su respuesta. También Israel (2014) halló que incluir comentarios sobre la relevancia de la pregunta resultaba en un mayor uso de palabras y de temas tanto en las encuestas en papel como en las encuestas online. En línea con estos resultados, Zuell et al. (2014), en una encuesta online con población universitaria, encontraron que la inclusión de comentarios motivacionales en las preguntas aumentaba la tasa de respuesta en preguntas abiertas, independientemente del tamaño del campo de texto.

\section{2.- Método}

\section{1.- Participantes}

El tamaño total de la muestra fue de 100 sujetos residentes en España. Las edades de los participantes estuvieron comprendidas entre los 19 y los 90 años, siendo su media de edad 45 años $(D T=20,36)$. El $54,1 \%$ fueron mujeres y el $45,9 \%$ hombres. Las principales características sociodemográficas desagregadas por grupos se presentan en la Tabla 1. Como se observa, no hubo diferencias significativas entre los grupos en sus principales características sociodemográficas, a excepción del nivel de ingresos $\left(X^{2}=\right.$ $19,39 ; p=, 02)$. Sin embargo, y a pesar de que no es una variable que según la investigación previa modere el efecto del tamaño del campo de respuesta, es preciso considerarla dada su relación con el nivel educativo $\left(\mathrm{r}_{\mathrm{s}}=, 29 ; \mathrm{p}<, 01\right)$. La muestra se extrajo por conveniencia, con aplicación de cuotas de edad [18-30 $(n=33), 31-50(n=$ $28), 51-70(n=23)$ y 71 o más $(n=16)]$.

\begin{tabular}{lcc} 
& $\begin{array}{c}\text { Modalidad A: } \\
\text { Texto pequeño } \\
\text { \% (n) }\end{array}$ & $\begin{array}{c}\text { Modalidad B: } \\
\text { Texto grande } \\
\text { \% (n) }\end{array}$ \\
\hline Edad $(\mathbf{D T})(t[98]=0,59 ; \mathrm{p}=, 55)$ & $46,52(21,69)$ & $44,10(19,09)$ \\
Sexo $\left(X^{2}[1]=0,37 ; p=, 54\right)$ & & \\
$\quad$ Mujer & $51,0 \%(25)$ & $57,1 \%(28)$ \\
$\quad$ Hombre & $49,0 \%(24)$ & $42,9 \%(21)$ \\
Nivel educativo $\left(X^{2}[2]=9,54 ; p=, 21\right)$ & & \\
$\quad$ Secundaria, Primaria o sin estudios & $12,2 \%(6)$ & $10,2 \%(5)$ \\
$\quad$ Bachillerato o FP & $49,0 \%(24)$ & $42,8 \%(21)$ \\
$\quad$ Estudios universitarios & $38,7 \%(19)$ & $46,9 \%(23)$ \\
Ingresos mensuales $\left(X^{2}[2]=19,39 ; p=, 02\right)$ & & $15,3 \%(6)$ \\
$\quad \leq 900 €$ & $19,6 \%(9)$ & $58,7 \%(27)$ \\
$901 €-3000 €$ & $67,4 \%(31)$ & $26,0 \%(12)$ \\
$>3000 €$ & $13,0 \%(6)$ &
\end{tabular}

Tabla 1. Características socio-demográficas por grupos 


\section{2.- Diseño de investigación e instrumento}

El estudio se ha implementado de acuerdo a un diseño split-ballot. Este diseño experimental responde a un procedimiento por el cual una muestra es dividida en dos mitades equivalentes, administrándose un cuestionario ligeramente diferente a cada una de ellas. La asignación de los sujetos a cada una de las mitades se ha efectuado mediante un criterio de asignación aleatoria dentro de los distintos grupos de edad. Los datos se recogieron a través de dos modalidades de un mismo cuestionario elaborado ad hoc para conocer la opinión de los ciudadanos respecto a temas relacionados con la justicia penal en España. La modalidad A del cuestionario presentaba tamaños de texto pequeños (9 milímetros), mientras que la modalidad B incorporaba tamaños de texto grandes (17 milímetros). La Figura 1 muestra la variación en el tamaño del campo de respuesta en ambas modalidades en una de las preguntas descriptivas recogidas en el cuestionario.

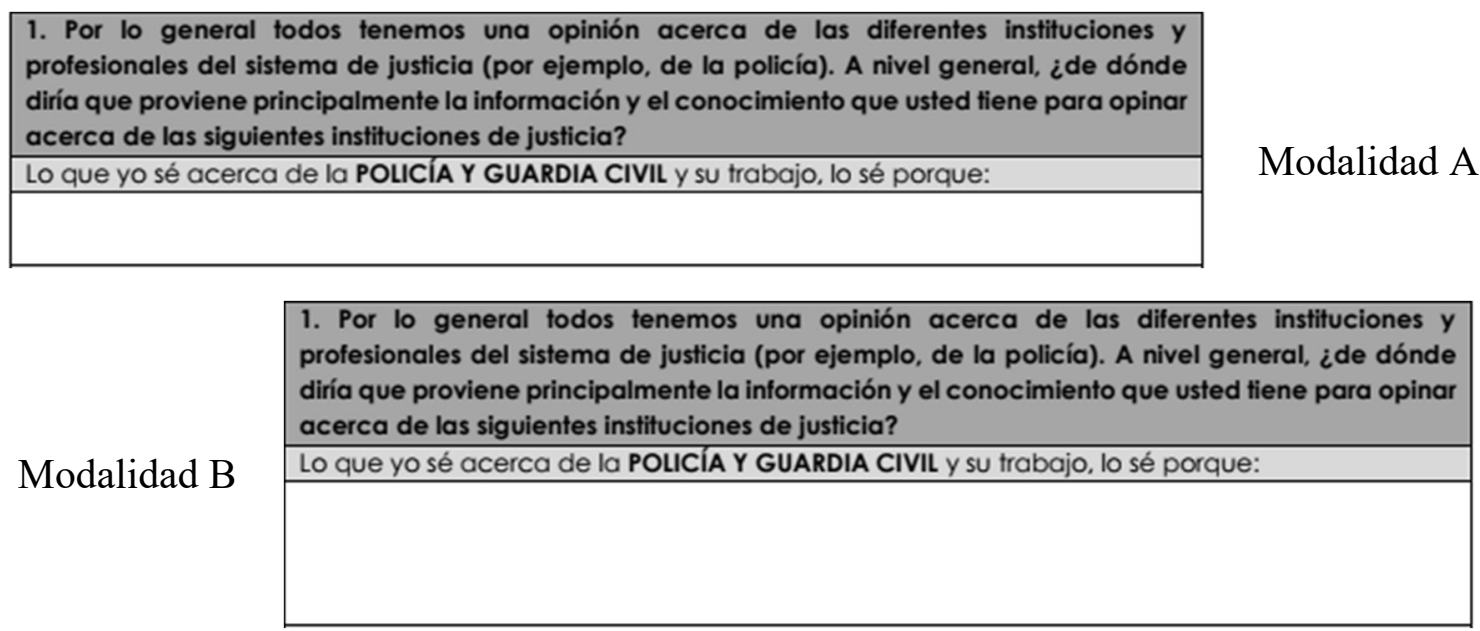

Figura 1. Variación en el tamaño del campo de texto en ambas modalidades.

El cuestionario constó de 16 preguntas abiertas de dos tipos: de listado (preguntas $1,2,7,8,9,10$ y 15) y descriptivas (preguntas $3,4,5,6,11,12,13,14$ y 16), así como diversas preguntas cerradas que no han sido objeto de este trabajo ${ }^{3}$. El contenido general de las preguntas hacía referencia a dos temas principales: la legitimidad social de las instituciones del sistema de justicia penal y las percepciones y actitudes hacia las víctimas de delitos.

\section{3.- Variables}

La variable dependiente de esta investigación fue el tamaño del campo de texto facilitado para las respuestas a preguntas abiertas. Una variable dicotómica cuyas categorías eran: grande (17 milímetros) y pequeño (9 milímetros).

Las variables independientes intervinientes en el estudio fueron el número de palabras, el número de temas y el tiempo de cumplimentación del cuestionario. El conteo del número de palabras se llevó a cabo con el programa Excel, a través de una fórmula que permitió contar el número de palabras sin tener en cuenta los espacios en blanco. Los temas fueron contabilizados manualmente una vez realizada la codificación cualitativa de las respuestas. Por último, para conocer el tiempo empleado por los encuestados en rellenar el cuestionario, se incluyeron dos preguntas, una al inicio y otra al final, en las que se preguntaba por la hora de comienzo y la hora de finalización del cuestionario. El 
tiempo empleado para rellenar el cuestionario fue calculado a través del asistente para hora y fecha del programa IBM-SPSS-Statistics.

\section{4.- Procedimiento}

Los datos se recogieron de forma individual mediante una encuesta autoadministrada en papel. Antes de proceder a la recogida de los datos utilizados para esta investigación se realizó una prueba piloto del cuestionario $(n=30)$ para comprobar su claridad y comprensión. Los cuestionarios fueron administrados durante los meses de enero a septiembre de 2017. El tiempo medio empleado para la cumplimentación del cuestionario fue de 27 minutos. No se ofrecieron incentivos por participar.

\section{5.- Análisis de datos}

Etapa 1. Codificación cualitativa de las respuestas a las preguntas abiertas. En un primer momento se llevó a cabo un análisis cualitativo de contenido dirigido a codificar las respuestas recogidas en las diversas preguntas abiertas del cuestionario. Este proceso consistió en la creación de una serie de temas o categorías a partir de la lectura cuidadosa de las respuestas recogidas en cada una de las preguntas abiertas de la encuesta (Richards, 2009; Silverman, 2011). De este modo, se fueron generando categorías que recogían los diferentes temas expresados en cada una de las respuestas y para cada una de las preguntas. Estas categorías fueron registradas en un libro de códigos, incluyendo una breve definición que permitía conocer cuándo aplicar cada uno de ellos. La codificación de los temas se realizó por dos investigadores distintos en función de la materia sobre la que versaban las preguntas, de tal forma que un investigador se encargó de la codificación de las respuestas a las preguntas sobre legitimidad social de las instituciones del sistema de justicia penal y otro investigador se ocupó de las respuestas sobre percepciones y actitudes hacia las víctimas. Posteriormente, las categorías fueron verificadas por un tercer investigador. La codificación realizada por el tercer investigador mostró un grado de acuerdo del $90,0 \%$ con las codificaciones realizadas previamente por los investigadores.

Etapa 2. Análisis cuantitativo de los datos. En primer lugar, se contabilizó tanto el número de palabras utilizadas por los encuestados para responder a cada una de las preguntas abiertas recogidas en el cuestionario, como el número de temas abordados en cada una de las respuestas. Posteriormente, se procedió al procesamiento y tratamiento estadístico de los datos utilizando el programa IBM-SPSS-Statistics en su versión 24.0. Se realizaron comparaciones de medias tanto del número de palabras como del número de temas para cada una de las respuestas proporcionadas en ambas modalidades de cuestionario. Para ello, atendiendo a las características de las variables, se utilizó el estadístico $t$ de Student y se calculó el tamaño de efecto utilizando la $d$ de Cohen. En consonancia con la literatura, tamaños menores de 0,3 se consideraron pequeños, tamaños cercanos a 0,5 fueron considerados medianos y tamaños superiores a 0,8 se consideraron grandes. También se analizaron las distribuciones de las preguntas que no tuvieron respuesta para ambas modalidades utilizando el estadístico Chi-cuadrado de Pearson y calculando el tamaño de efecto con la $V$ de Cramer. Se utilizó el estadístico $t$ de Student para comparar el número total de preguntas sin respuesta en ambas modalidades. Finalmente, se comparó el tiempo medio de cumplimentación del cuestionario para ambas modalidades mediante el estadístico $t$ de Student. 


\section{3.- Resultados}

\section{1.- ¿Cómo Afecta el Tamaño del Campo de Texto al Número de Palabras Utilizadas por los Encuestados en sus Respuestas?}

Para comprobar la primera hipótesis se comparó la media de palabras empleadas por los participantes en sus respuestas a las distintas preguntas incluidas en el cuestionario para ambas modalidades. Como se muestra en la Tabla 2, a excepción de lo que ocurrió en la pregunta 15, en el resto de preguntas abiertas la media de palabras fue superior en los cuestionarios que contenían campos de texto grandes. La diferencia en el número medio de palabras entre ambas modalidades osciló entre 0,70 palabras para la pregunta 10 y 7,67 palabras para la pregunta 11. Además, fueron halladas diferencias significativas entre las modalidades en 10 de las 16 preguntas incluidas en el cuestionario (esto es, en el $62,5 \%$ de los casos). Tomados en conjunto, estos resultados respaldan la hipótesis planteada al mostrar que, para la mayoría de las preguntas incluidas en el cuestionario, la media de palabras utilizadas en las respuestas fue superior en la modalidad de cuestionario que utilizaba campos de texto grandes (modalidad B) en comparación con la modalidad que utilizaba campos de texto pequeños (modalidad A).

Igualmente cabe mencionar dos aspectos relevantes. En primer término, si se profundiza en el tipo de preguntas que presentaron diferencias significativas entre modalidades, se observa que el tamaño del campo de texto afectó a las respuestas de los encuestados independientemente de la naturaleza de las preguntas (descriptivas o de listado). En este sentido, y como muestra la Tabla 2, se encontraron diferencias significativas entre las modalidades tanto en preguntas de naturaleza descriptiva (preguntas 3, 4, 5, 6, 11, 13 y 16), como en preguntas de listado (preguntas 1, 2 y 7). Sin embargo, fue mayor el número de preguntas de tipo descriptivo afectadas por el tamaño del campo de texto $(77,8 \%$ versus $42,9 \%)$. Además, el tamaño de efecto también fue superior en este tipo de preguntas. De este modo, mientras que las preguntas tipo listado mostraron un tamaño de efecto pequeño-mediano, las preguntas de naturaleza descriptiva presentaron tamaños de efecto medianos y grandes (ver Tabla 2).

En segundo término, y aunque no se manipuló experimentalmente la ubicación de las preguntas dentro del cuestionario, se encontró que el tamaño del campo de texto afectó a las respuestas de los participantes independientemente de su localización. De este modo, considerando las preguntas que presentaron diferencias significativas entre las modalidades, se observó que a pesar de que estas diferencias se hallaron principalmente en las preguntas situadas al inicio del cuestionario (preguntas 1-7), el tamaño del efecto fue mayor en las preguntas colocadas al final del mismo (preguntas 11, 13 y 16). Así, mientras que las preguntas situadas al inicio del cuestionario mostraron tamaños de efecto medianos $(d$ de Cohen $\approx 0,50)$, las preguntas situadas al final mostraron tamaños de efecto cercanos a grandes $(d$ de Cohen $\approx 0,80)$ (ver Tabla 2$)$. 


\begin{tabular}{|c|c|c|c|c|c|}
\hline $\begin{array}{l}\text { Número y tipo } \\
\text { de pregunta }\end{array}$ & $\begin{array}{c}\text { Modalidad A } \\
\text { Texto pequeño } \\
\bar{x} \text { palabras }\end{array}$ & $\begin{array}{c}\text { Modalidad B } \\
\text { Texto grande } \\
\bar{x} \text { palabras }\end{array}$ & $\begin{array}{l}\text { Diferencia de } \\
\text { medias }\end{array}$ & $t$ & d de Cohen \\
\hline 1 (L) & 7,06 & 9,94 & 2,88 & $-2,08^{*}$ & $-0,41$ \\
\hline 2 (L) & 4,86 & 8,24 & 3,38 & $-2,68 * *$ & $-0,53$ \\
\hline 3 (D) & 7,10 & 12,04 & 4,94 & $-2,38 *$ & $-0,48$ \\
\hline 4 (D) & 6,02 & 11,84 & 5,82 & $-2,29 *$ & $-0,46$ \\
\hline 5 (D) & 8,02 & 12,47 & 4,45 & $-2,42 *$ & $-0,48$ \\
\hline 6 (D) & 6,88 & 11,60 & 4,72 & $-3,25 * *$ & $-0,65$ \\
\hline 7 (L) & 4,12 & 7,86 & 3,74 & $-2,53 *$ & $-0,51$ \\
\hline 8 (L) & 4,55 & 5,44 & 0,89 & $-0,91$ & $-0,18$ \\
\hline 9 (L) & 3,02 & 4,30 & 1,28 & $-1,60$ & $-0,32$ \\
\hline $10(\mathrm{~L})$ & 3,02 & 3,72 & 0,70 & $-0,98$ & $-0,20$ \\
\hline 11 (D) & 7,64 & 15,31 & 7,67 & $-3,54 * *$ & $-0,74$ \\
\hline 12 (D) & 6,52 & 7,84 & 1,32 & $-1,24$ & $-0,24$ \\
\hline 13 (D) & 5,32 & 9,41 & 4,09 & $-3,34 * *$ & $-0,67$ \\
\hline 14 (D) & 11,55 & 13,38 & 1,83 & $-0,95$ & $-0,19$ \\
\hline $15(\mathrm{~L})$ & 5,12 & 5,00 & $-0,12$ & 0,14 & 0,02 \\
\hline 16 (D) & 7,06 & 12,82 & 5,76 & $-3,80 * *$ & $-0,78$ \\
\hline
\end{tabular}

Nota: $\mathrm{L}=$ Pregunta de tipo listado de ítems; $\mathrm{D}=$ Pregunta de tipo descriptivo

$* \alpha \leq, 05: * * \alpha \leq, 01$

Tabla 2. Comparación de medias en el número de palabras por pregunta y modalidad.

3.2.- ¿Cómo Afecta el Tamaño del Campo de Texto al Número de Temas Desarrollados por los Encuestados en sus Respuestas?

Para comprobar si el tamaño del campo de texto también afectó al número de temas abordados por los participantes en sus respuestas, se realizó una comparación de medias del número de temas desarrollados por los encuestados en sus respuestas a las distintas preguntas incluidas en el cuestionario para ambas modalidades. Como se muestra en la Tabla 3, 9 de las 16 preguntas incluidas en el cuestionario, esto es, el 56,3\%, presentaron un mayor número medio de temas en la modalidad con los tamaños grandes del campo de texto. Sin embargo, estas diferencias entre modalidades fueron pequeñas y, en general, no alcanzaron la significación estadística. De este modo, solo fueron halladas diferencias significativas en la pregunta $11(t[92]=-2,17 ; p=, 03 ; d=-0,44)$ y diferencias marginalmente significativas en la pregunta $7(t[96]=-1,89 ; p=, 06 ; d=-0,39)$. En ambos casos el tamaño de efecto fue pequeño-mediano.

\begin{tabular}{|c|c|c|c|c|c|}
\hline $\begin{array}{l}\text { Número y tipo } \\
\text { de pregunta }\end{array}$ & $\begin{array}{c}\text { Modalidad A } \\
\text { Texto pequeño } \\
\bar{x} \text { temas }\end{array}$ & $\begin{array}{c}\text { Modalidad B } \\
\text { Texto grande } \\
\bar{x} \text { temas }\end{array}$ & $\begin{array}{l}\text { Diferencia de } \\
\text { medias }\end{array}$ & $t$ & $\begin{array}{c}\text { d de } \\
\text { Cohen }\end{array}$ \\
\hline 1 (L) & 1,46 & 1,48 & 0,02 & $-0,16$ & $-0,03$ \\
\hline $2(\mathrm{~L})$ & 1,22 & 1,32 & 0,10 & $-1,06$ & $-0,21$ \\
\hline 3 (D) & 1,95 & 2,04 & 0,09 & $-0,40$ & $-0,09$ \\
\hline 4 (D) & 1,79 & 1,78 & $-0,01$ & 0,06 & 0,01 \\
\hline 5 (D) & 1,81 & 1,63 & $-0,18$ & 1,05 & 0,21 \\
\hline 6 (D) & 1,54 & 1,60 & 0,06 & $-0,41$ & $-0,08$ \\
\hline 7 (L) & 1,33 & 1,60 & 0,27 & $-1,89^{\dagger}$ & $-0,39$ \\
\hline 8 (L) & 1,10 & 1,26 & 0,16 & $-1,46$ & $-0,30$ \\
\hline 9 (L) & 1,14 & 1,20 & 0,06 & $-0,61$ & $-0,14$ \\
\hline $10(\mathrm{~L})$ & 1,06 & 1,04 & $-0,02$ & 0,45 & 0,09 \\
\hline 11 (D) & 1,11 & 1,36 & 0,25 & $-2,17 *$ & $-0,44$ \\
\hline 12 (D) & 1,52 & 1,46 & $-0,06$ & 0,15 & 0,03 \\
\hline 13 (D) & 1,14 & 1,14 & 0,00 & $-0,04$ & 0,00 \\
\hline 14 (D) & 1,61 & 1,46 & $-0,15$ & 1,16 & 0,22 \\
\hline $15(\mathrm{~L})$ & 2,93 & 2,71 & $-0,22$ & 0,87 & 0,17 \\
\hline 16 (D) & 1,65 & 1,93 & 0,28 & 0,81 & $-0,32$ \\
\hline
\end{tabular}

Nota: $\mathrm{L}=$ Pregunta de tipo listado de ítems; $\mathrm{D}=$ Pregunta de tipo descriptivo

$* \alpha<, 05: \dagger \alpha \leq, 10$

Tabla 3. Comparación de medias en el número de temas por pregunta y modalidad. 


\section{3.- ¿Cómo Afecta el Tamaño del Campo de Texto al Número de Preguntas sin Respuesta?}

Con la finalidad de contrastar la tercera hipótesis se compararon y analizaron aquellas preguntas que no habían obtenido respuesta para cada una de las modalidades de cuestionario. Tras aplicar una prueba $t$ para muestras independientes, los resultados mostraron que existen diferencias marginalmente significativas entre las modalidades en el número total de respuestas en blanco $(t[98]=1,98 ; p=, 05 ; d=0,39)$. En promedio, los participantes dejaron sin respuesta aproximadamente una pregunta en la modalidad que presentó campos pequeños de texto $(M=1,02 ; D T=2,19)$, mientras que esta cifra se redujo a $0,36(D T=0,85)$ en la modalidad con campos grandes de texto. Sin embargo, a pesar de esta diferencia en el número total de preguntas sin respuesta a lo largo del cuestionario, los resultados no mostraron diferencias significativas entre las modalidades para cada una de las preguntas (ver Figura 2). En este sentido, solo fueron halladas diferencias marginalmente significativas en las preguntas $14\left(X^{2}=3,09 ; p=, 07 ; V=0,17\right)$ y $16\left(X^{2}=3,09 ; p=, 07 ; V=0,17\right)$. En ambos casos las preguntas fueron descriptivas y los tamaños de efecto pequeños.

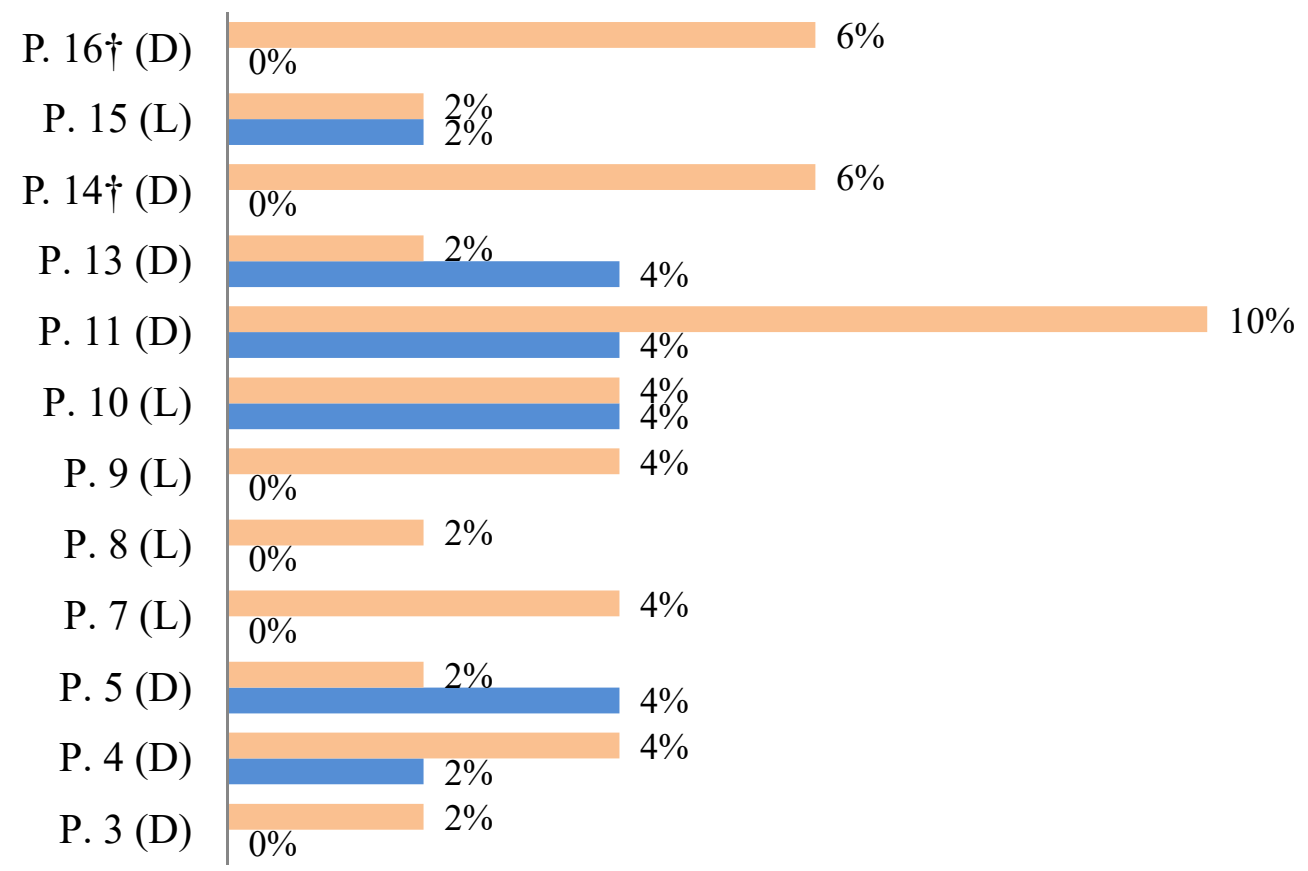

$\square$ Modalidad A (Campo de texto pequeño) $\quad$ Modalidad B (Campo de texto grande)

Notas: Las preguntas no recogidas en la figura no tuvieron preguntas sin respuesta.

$\mathrm{L}=$ Pregunta de tipo listado de ítems; $\mathrm{D}=$ Pregunta de tipo descriptivo

$\dagger \alpha \leq, 10$

Figura 2. Porcentaje de no respuesta por pregunta y modalidad.

3.4.- ¿Afecta el Tamaño del Campo de Texto al Tiempo Medio Empleado por los Encuestados para Rellenar el Cuestionario?

Por último, se comparó el tiempo medio empleado por los participantes para rellenar los cuestionarios en ambas modalidades. Los resultados mostraron que los tiempos medios fueron muy similares en ambas modalidades, siendo la diferencia de 8 
segundos (0:27:03 versus 0:26:55). Además, tras aplicar una prueba $t$ para muestras independientes, los resultados confirmaron que no existieron diferencias significativas entre las modalidades en el tiempo de cumplimentación del cuestionario $(t[99]=0,04 ; p$ $=, 96)$.

\section{4.- Discusión.}

\section{1.- ¿Cómo Afecta el Tamaño del Campo de Texto al Número de Palabras Utilizadas por los Encuestados en sus Respuestas?}

En primer lugar, cabe recalcar que, para la mayoría de las preguntas incluidas en los análisis, la media de palabras empleadas por los encuestados en sus respuestas fue superior en la modalidad que presentó campos de texto grandes, en comparación con la modalidad con campos de texto pequeños. Estos hallazgos son consistentes con los de otras investigaciones que han encontrado que las respuestas de los encuestados son más extensas cuando el espacio facilitado para la respuesta es de mayor tamaño (Christian \& Dillman, 2004; Israel, 2010; Maloshonok \& Terentev, 2016; Stern et al., 2007).

Además, se encontró que tamaño del campo de respuesta afectaba especialmente a las preguntas de naturaleza descriptiva. Estos resultados vendrían avalados por estudios previos que han hallado que el tamaño del campo de texto influye especialmente en la cantidad de información que los encuestados proporcionan a preguntas que requieren una respuesta narrativa, mientras que las preguntas de listado o elementos numéricos parecen verse menos afectadas por el tamaño del campo de respuesta proporcionado (Couper et al., 2011; Keusch, 2014). Probablemente porque, mientras que las primeras requieren un mayor nivel de redacción en su respuesta, las segundas pueden ser contestadas con un desarrollo menor.

Por otro lado, y a pesar de no haberse hipotetizado sobre ello, se advirtió que el tamaño del campo de texto tenía mayores efectos en las preguntas situadas al final del cuestionario, lo que podría explicarse por la mayor fatiga de los encuestados. Sin embargo, y a pesar de que el efecto puede haberse acentuado por esta razón, en este estudio solo se manipuló el tamaño del campo de texto y no es posible precisar la contribución de esta característica a los resultados obtenidos. En consecuencia, se propone que futuros estudios manipulen experimentalmente esta variable para conocer su influencia e interacción con el tamaño del campo de respuesta.

\section{2.- ¿Cómo Afecta el Tamaño del Campo de Texto al Número de Temas Desarrollados por los Encuestados en sus Respuestas?}

El análisis del número medio de temas abordados por los encuestados en sus respuestas mostró que, si bien el número medio era superior en la modalidad que empleó campos grandes de texto, los resultados no fueron en general significativos. Esto podría explicarse por el interés expresado por los participantes en el tema de la encuesta, ya que el $67,0 \%$ de los encuestados señaló tener bastante o mucho interés, lo que podría reducir los efectos de la manipulación en el campo de texto (Holland \& Christian, 2009).

Atendiendo a la naturaleza de las preguntas, la única diferencia significativa entre modalidades se encontró en una pregunta de tipo descriptivo ("Ahora piense en todas las experiencias que recuerde, o las más significativas, que ha tenido a lo largo de su vida con diferentes instituciones y agentes del sistema de justicia penal -como la policía, guardia civil, tribunales penales, etc.-. Díganos brevemente cuál fue el motivo de ese contacto, de esa experiencia con las autoridades). Considerando el objeto de la pregunta, que busca conocer las experiencias que los encuestados han tenido con las distintas 
instituciones y agentes del sistema de justicia penal, parece lógico que las respuestas proporcionadas incorporasen un mayor número de temas en la modalidad con los tamaños grandes del campo de texto. En este sentido, los encuestados, en su pretensión por ofrecer una respuesta que rellene el espacio del campo de texto proporcionado (Emde \& Fuchs, 2012), podrían haber incluido un mayor número de experiencias y, por tanto, de temas, en la modalidad con los campos de texto grandes. Sin embargo, esto no ocurriría en la modalidad que presentó tamaños pequeños del campo de texto, cuyo espacio de respuesta podría completarse con un menor número de experiencias y, en consecuencia, de temas.

Por otro lado, el hecho de que la única diferencia significativa entre las modalidades se presente en una pregunta situada al final del cuestionario parece confirmar que los efectos del tamaño del campo de texto se acentúan en las preguntas recogidas al final del mismo. Sin embargo, y como se apuntaba en líneas anteriores, futuros estudios podrán manipular experimentalmente la ubicación de las preguntas para conocer qué influencia concreta ejerce este factor.

\section{3.- ¿Cómo Afecta el Tamaño del Campo de Texto al Número de Preguntas sin Respuesta?}

Los datos resultantes respaldaron parcialmente la hipótesis planteada al mostrar que, a nivel global, el número de respuestas en blanco fue superior en la modalidad con campos pequeños de texto, aunque la diferencia fue marginalmente significativa. En este sentido, la mayor sobrecarga que recaería sobre el encuestado al utilizar campos de texto grandes no se tradujo en un aumento de la ausencia de respuestas. Estos resultados están en sintonía con una parte de la literatura previa que ha hallado que el tamaño del campo de texto no afecta de forma significativa a la tasa de no respuesta en cuestionarios autoadministrados con preguntas abiertas como el empleado en este trabajo (Christian \& Dillman, 2004; Israel, 2006, 2010; Smyth et al., 2009). No obstante, estos resultados parecen no reproducirse en las encuestas online, donde diversos autores han encontrado un aumento significativo de la tasa de no respuesta cuando se empleaban tamaños grandes del campo de texto (Emde \& Fuchs, 2012; Keusch, 2014; Scholz \& Zuell, 2012; Zuell et al., 2014). Futuros estudios podrán profundizar en este aspecto analizando la influencia de la forma de administración del cuestionario y el dispositivo desde el que se contesta (por ejemplo, móvil, ordenador o tableta) en la cantidad de preguntas sin respuesta.

Por otro lado, los resultados hallados muestran que el efecto del tamaño del campo de texto en el número de preguntas que no han obtenido respuesta se produce únicamente en preguntas de naturaleza descriptiva que están ubicadas al final del cuestionario (preguntas 14 y 16). Otro aspecto destacable a nivel general es la baja tasa de preguntas en blanco en el cuestionario (en torno al 3,0\%). Estos resultados podrían explicarse por el elevado interés indicado por los participantes de ambas modalidades en el tema de la encuesta. En este sentido, el 66,0\% de los encuestados en la modalidad A y el 68,0\% de los encuestados en la modalidad B señalaron bastante o mucho interés en el tema. Además, no se hallaron diferencias significativas entre las modalidades en esta variable $\left(X^{2}=4,33 ; p=, 22\right)$. Futuras investigaciones podrán profundizar en estos resultados analizando las diferencias en la tasa de respuesta a las preguntas entre quienes muestran un elevado interés en el tema y quienes muestran un interés bajo

\section{4.- ¿Afecta el Tamaño del Campo de Texto al Tiempo Medio Empleado por los Encuestados para Rellenar el Cuestionario?}

Los resultados obtenidos no permitieron respaldar la hipótesis de que los campos de texto grandes aumentan el tiempo de respuesta del cuestionario al tener que proporcionar respuestas más elaboradas. Sin embargo, están en sintonía con el estudio 
realizado por Couper et al. (2011), quienes tampoco encontraron diferencias en el tiempo de respuesta según el tamaño del campo de texto utilizado. No obstante, es preciso señalar que la literatura no se ha mostrado unánime en este aspecto. De esta forma, si bien ciertos estudios no han encontrado diferencias en el tiempo de respuesta según el tamaño del campo de texto utilizado, otros sí lo han hecho. Este es el caso del estudio realizado por Keusch (2014) quien, a través de un estudio experimental donde manipuló el tamaño y el número de campos de respuesta, encontró que los encuestados empleaban un tiempo significativamente superior cuando la respuesta se proporcionaba en diez campos de texto pequeños, en comparación con un solo campo de texto grande. Sin embargo, este estudio se realizó mediante una encuesta online compuesta por preguntas abiertas de tipo listado, lo que podría haber influido también sobre el efecto del campo de texto y sobre el tiempo empleado en contestar a las preguntas. Además, debe tenerse en cuenta en la interpretación de los resultados dos aspectos relevantes; en primer término, Keusch (2014) midió el tiempo de respuesta a las preguntas de manera individual, no el tiempo total de cumplimentación del cuestionario. En segundo lugar, es importante señalar que mientras que en las encuestas online el tiempo de cumplimentación es un dato objetivo (paradata), en el presente estudio, al tratarse de un cuestionario en papel, el tiempo de cumplimentación fue indicado por los propios encuestados, por lo que está sujeto a un mayor error. Futuras investigaciones podrán profundizar en este aspecto analizando la influencia e interacción entre estos factores.

\section{5.- Conclusiones}

Investigaciones previas han sugerido que el diseño visual del cuestionario afecta a las respuestas de los encuestados y, por tanto, a la calidad de los datos obtenidos, tanto en preguntas cerradas como en preguntas abiertas. Sin embargo, y a pesar de la importancia y utilidad de estos estudios en la orientación práctica del diseño de cuestionarios, los estudios realizados en el contexto español sobre cómo afecta el tamaño del campo de texto a las respuestas de los encuestados son escasos. Por ello, y con el propósito de contribuir al desarrollo de este campo de estudio, el presente trabajo ofrece información relevante sobre el efecto de manipular el tamaño del campo de respuesta en un cuestionario auto-administrado en papel. Además, resulta novedoso dentro de este ámbito de trabajo gracias a la inclusión de preguntas de distinta naturaleza (descriptivas y de listado) y al análisis de cuatro indicadores relacionados con las respuestas como son el número de palabras, el número de temas, la proporción de preguntas sin respuesta y el tiempo empleado para cumplimentar el cuestionario.

Atendiendo a los resultados obtenidos se llega principalmente a tres conclusiones. En primer término, se constata que el tamaño del campo de texto afecta al número medio de palabras empleadas por los encuestados en sus respuestas. Además, los resultados también sugieren que los efectos del tamaño del campo de texto se intensifican cuando las preguntas están situadas al final del cuestionario y cuando estas preguntas son de tipo descriptivo. No obstante, el aumento significativo en el número de palabras que se observa en el cuestionario con campos de texto grandes no se traduce, en general, en un mayor número de temas. De este modo, los encuestados que recibieron cuestionarios con campos de texto mayores parecen elaborar en mayor medida sus temas, pero no aportar temas adicionales. Tomados en conjunto, estos resultados sugieren que el diseño visual influye en las respuestas de los encuestados, por lo que debe ser tenido en cuenta a la hora de diseñar cuestionarios. 
En segundo lugar, los resultados muestran que el tamaño del campo de texto no afecta significativamente al porcentaje de respuestas en blanco. Futuros estudios podrán profundizar en este sentido analizando la posible relación entre el interés de los encuestados en el tema y el porcentaje de preguntas sin respuesta. Además, se propone como línea de investigación futura analizar los efectos de incluir comentarios motivacionales a lo largo del cuestionario sobre el porcentaje de preguntas sin respuesta en cuestionarios con diferentes tamaños del campo de respuesta.

Finalmente, los resultados indican que el tiempo empleado por los encuestados para rellenar el cuestionario fue muy similar en ambas modalidades. No obstante, estos resultados parecen ser diferentes cuando se trata de encuestas online. Por ello, futuras investigaciones podrán analizar si el modo en el que se administran los cuestionarios repercute en el tiempo medio de cumplimentación y si los efectos del campo de texto se acentúan cuando los cuestionarios son administrados online en comparación con otros procedimientos.

\section{6.- Notas}

[1] La expresión campo de texto hace referencia al espacio proporcionado para recoger las respuestas de los encuestados.

[2] Smyth et al. (2009) emplearon el término de encuestados "precoces" para referirse a aquellos participantes que habían cumplimentado el cuestionario en el mismo plazo o antes que el 50,0\% de los encuestados. Por el contrario, emplearon el término de encuestados "tardíos" para referirse a aquellos que cumplimentaron el cuestionario más tarde que el $50 \%$ de los participantes.

[3] El cuestionario estuvo compuesto por un total de treinta y seis preguntas. Entre ellas, además de las dieciséis preguntas abiertas que fueron objeto de estudio, se incluyeron diez preguntas referidas a aspectos sociodemográficos de los encuestados, dos cuestiones relativas a la hora de inicio y finalización del cuestionario y una pregunta sobre el interés general de los participantes en el tema de la encuesta. Las siete preguntas restantes fueron

\section{7.- Referencias}

Barrios, M., Villarroya, A., Borrego, A., \& Ollé, C. (2010). Response rates and data quality in web and mail surveys administered to $\mathrm{PhD}$ holders. Social Science Computer Review, 29, 208-220. doi: 10.1177/0894439310368031

Chaudhary, A. K. \& Israel, G. D. (2016). Influence of importance statements and box size on response rate and response quality of open-ended questions in web/mail mixedmode surveys. Journal of Rural Social Sciences, 31, 140-159.

Christian, L. M. \& Dillman, D. A. (2004). The influence of graphical and symbolic language manipulations on responses to self-administered questions. Public Opinion Quarterly, 68, 57-80. doi: 10.1093/poq/nfh004

Christian, L. M., Dillman, D. A., \& Smyth, J. D. (2007). Helping the respondents get it right the first time: the influence of words, symbols, and graphics in Web surveys. Public Opinion Quarterly, 71, 113-125. doi: 10.1093/poq/nfl039 
Couper, M. P., Conrad, F. G., \& Tourangeau, C. R. (2007). Visual context effects in web surveys. Public Opinion Quarterly, 4, 623-634. doi:10.1093/poq/nfm044

Couper, M. P., Kennedy, C., Conrad, F. G., \& Tourangeau, R. (2011). Designing input fields for non-narrative open-ended responses in web surveys. Journal of Official Statistics, 27, 65-85.

Denscombe, M. (2008). The length of responses to open-ended questions. A comparison of online and paper questionnaires in terms of a mode effect. Social Science Computer Review, 26, 359-368. doi: 10.1177/0894439307309671

Deutskens, E., de Ruyter, K., \& Wetzels, M. (2006). An assessment of equivalence between online and mail surveys in service research. Journal of Service Research, 8, 345-355. doi: 10.1177/1094670506286323

Díez-Ripollés, J. L. \& García-España, E. (2009). Encuesta a víctimas en España. Málaga: Instituto Andaluz Interuniversitario de Criminología.

Dillman, D. A., Smyth, J. D., \& Christian, L. M. (2009). Internet, mail, and mixed-mode surveys. The tailored design method (3rd Ed.). Hoboken, NJ: Wiley

Emde, M. \& Fuchs, M. (2012). Using adaptive questionnaire design in open-ended questions: A field experiment. Paper presented at the American Association for Public Opinion Research (AAPOR) 67th Annual Conference, San Diego, USA.

Fuchs, M. (2009). Differences in the visual design language of paper-and-pencil surveys versus web surveys: A field experimental study on the length of response fields in open-ended frequency questions. Social Science Computer Review, 27, 213-227. doi: $10.1177 / 0894439308325201$

Groves, R. M., Fowler, F. J., Couper, M. P., Lepkowski, J. M., Singer, E., \& Tourangeau, R. (2009). Survey methodology ( $2^{\text {nd }}$ Ed.). Hoboken, New Jersey: Wiley.

Hedberg, E. C., Wallace, D., \& Cesar, G. (2013). The effect of survey mode on socially undeirable responses to open ended questions: online vs. paper instruments. Paper presented at the Annual Conference of American Association for Public Opinion Research, Boston.

Holland, J. L. \& Christian, L. M. (2009). The influence of topic interest and interactive probing on responses to open-ended questions in web surveys. Social Science Computer Review, 27, 197-212. doi: 10.1177/0894439308327481

Israel, G. D. (2006). Visual cues and response format effects in mail surveys. Paper presented at the Annual Meeting of the Southern Rural Sociological Association, Orlando, FL.

Israel, G. D. (2010). Effects of answer space size on responses to open-ended questions in mail surveys. Journal of Official Statistics, 26, 271-285. 
Israel, G. D. (2014). Using motivating prompts to increase responses to open-ended questions in mixed-mode surveys: Evidence on where the prompt should be placed. Paper presented at the annual conference of the American Association for Public Opinion Research (AAPOR), Anaheim, CA.

Keusch, F. (2014). The influence of answer box format on response behavior on list-style open-ended questions. Journal of Survey Statistics and Methodology, 2, 305-322. doi: $10.1093 /$ jssam/smu007

Krosnick, J. A. \& Presser, S. (2009). Question and questionnaire design. In Marsden, P.V. \& Wright, J.D. (Eds.). The handbook of survey research (pp. 263-314). Bingley, UK: Emerald Group Publising.

Kwak, N. \& Radler, B. (2002). A comparison between mail and web surveys: Response pattern, respondent profile, and data quality. Journal of Official Statistics, 18, $257-273$.

MacElroy, B., Mikucki, J., \& McDowell, P. (2002). A comparison of quality in open-end responses and response rates between web-based and paper and pencil survey modes. Journal of Online Research. Available at http://www.websm.org/uploadi/editor/comparison.pdf

Maloshonok, N. \& Terentev, E. (2016). The impact of visual design and response formats on data quality in a web survey of MOOC students. Computers in Human Behavior, 62, 506-515. doi: 10.1016/j.chb.2016.04.025

Mohr, A., Sell, A., \& Lindsay, T. (2015). Thinking inside the box: Visual design of the response box affects creative divergent thinking in an online survey. Social Science Computer Review, 1-13. doi: $10.1177 / 0894439315588736$

Richards, L. (2009). Handling qualitative data: A practical guide. London: SAGE.

Scholz, E. \& Zuell, C. (2012). Item non-response in open-ended questions: Who does not answer on the meaning of left and right? Social Science Research, 41, 1415-1428.

Silverman, D. (2011). Interpreting qualitative data. London: SAGE.

Smith, T. W. (1993). Little things matter: A sampler of how differences in questionnaire format can affect survey responses. GSS Methodological Report, 78. Chicago: National Opinion Research Center.

Smyth, J. D., Dillman, D. A., Christian, L. M., \& McBride, M. (2009). Open-ended questions in web surveys. Can increasing the size of answer boxes and providing extra verbal instructions improve response quality? Public Opinion Quarterly, 73, $325-337$.

Stern, M. J., Dillman, D. A., \& Smyth, J. D. (2007). Visual design, order effects, and respondent characteristics in a self-administered survey. Survey Research Methods, 1, 121-138. 
Tourangeau, R., Couper, M. P., \& Conrad, F. G. (2004). Spacing, position, and order: Interpretive heuristics for visual features of survey questions. Public Opinion Quarterly, 68, 368-393.

Zuell, C., Menold, N., \& Körber, S. (2014). The influence of the answer box size on item nonresponse to open-ended questions in a web survey. Social Science Computer Review, 33, 115-122. doi: 10.1177/0894439314528091 